\title{
argestre
}

\section{Uma ferramenta para aplicação de mapeamentos entre termos SNOMED CT, CID-10 e CIAP-2 e enriquecimento terminológico em Segundas-Opiniões Formativas sobre hipertensão e diabetes}

\begin{abstract}
A tool to enable mappings among SNOMED CT, ICD-10 and ICPC-2 terms and terminological enrichment on Second-opinions about hypertension and diabetes
\end{abstract}

\author{
Bruno Gomes Tavares dos Santos ${ }^{1}$, Giovani Nícolas Bettoni ${ }^{2}$, Filipe Santana da \\ Silva $^{3}$
}

\section{Resumo}

Introdução: Um Sistema de Registro Eletrônico em Saúde (S-RES) requer que sejam utilizadas terminologias e vocabulários controlados (como a SNOMED-CT, CID-10 e CIAP2) para garantir a interoperabilidade semântica entre sistemas. Todavia, nem todos os $S$ RES incorporam esse tipo de abordagem, minimizando a utilização das fontes terminológicas para a codificação de documentos clínicos. Objetivo: O objetivo deste trabalho é apresentar uma ferramenta que permita explorar os mapeamentos existentes entre CID-10, CIAP-2 e SNOMED CT para indicação de termos correlacionados para enriquecimento terminológico em SOFS. Método: Nossa abordagem permite recuperar SOFs diretamente da BVS APS utilizando Web scraping e a construção de uma solução baseada em bancos de dados para sugerir mapeamentos provenientes de fontes consolidadas, facilitando a consulta e a recuperação de novos códigos da CID-10 e da SNOMED CT. Resultados: O resultado foi a disponibilização de uma estratégia simples para explorar mapeamentos entre os termos das três fontes terminológicas. Conclusão: Como conclusão, esta abordagem pode ser incorporada para a sugestão de novos termos na codificação de SOFS ou de S-RES num contexto mais amplo. Além disso, trabalhos futuros nestes mapeamentos são necessários para expansão para outros módulos importantes na atenção primária.

Palavras-Chave: S-RES. SNOMED-CT. CID-10. CIAP-2. SOF. Interoperabilidade. Hipertensão. Diabetes

\footnotetext{
Abstract

Introduction: An Electronic Health Record System (EHR-S) requires that usage of controlled terminology and vocabulary (such as SNOMED-CT, ICD-10, and ICPC-2) to ensure semantic interoperability. However, not all EHR-S incorporate such an approach, minimizing the use of terminological sources for clinical document coding. Objective: The

1 Discente. Bacharelado em Informática Biomédica. Universidade Federal de Ciências da Saúde de Porto Alegre. Email: brunogt@ufcspa.edu.br

2 Discente. Bacharelado em Informática Biomédica. Universidade Federal de Ciências da Saúde de Porto Alegre. Email: giovanib@ufcspa.edu.br

3 Doutor em Ciência da Computação. Departamento de Ciências Exatas e Sociais Aplicadas (DECESA), Universidade Federal de Ciências da Saúde de Porto Alegre. ORCID: 0000-0002-6803-1407. Email: filipe@ufcspa.edu.br

*Correspondência: Rua Sarmento Leite, 245, Sala 400C, Centro, Porto Alegre, Brasil. CEP: 90050-170.
}

Rev. Saúde Digital Tec. Educ., Fortaleza, CE, v. 5, n. 1, p.95-107, jan./abr. 2020.

ISSN: 2525-9563 
objective of this work was to present a tool that allows exploring existing terminological mappings among ICD-10, ICPC-2 and SNOMED CT for the indication of correlated terms for terminological enrichment on Second opinions (SOFs). Method: Our approach enables the retrieval of SOFs directly from the BVS APS using Web scraping as well as the construction of a solution based on a database of mappings taken from consolidated sources, enabling queries and retrieval of new ICD-10 and SNOMED CT codes. Results: The result was the delivery of a simple approach for exploring mappings between terms of these terminological sources programmatically. Conclusion: As a conclusion, this work can be directly incorporated with SOFs or in a EHR-S to suggest new terms and to enable broader encoding for clinical documents. Furthermore, future works on these mappings are necessary for the expansion of other important modules on primary healthcare.

Keywords: EHR-S. SNOMED-CT. ICD-10. ICPC-2. Interoperability. Second-opinion. Hypertension. Diabetes.

\section{Introdução}

Um Sistema de Registro Eletrônico em Saúde (S-RES) é definido como um conjunto de informações sobre um (ou mais) indivíduo (s) em um formato eletrônico, de modo a garantir um cuidado a saúde de qualidade e permitir que exista troca de informações segura sobre a saúde deste, entre profissionais da saúde (1). Diferentemente do Prontuário Eletrônico do Paciente (PEP), um S-RES é mais genérico e incorpora outras informações, como, por exemplo o contexto de saúde, prescrição, procedimentos, resultados de exames e laudos, dados demográficos, entre outros (2) (3).

Um dos motivos pelo qual os S-RES estão sendo cada vez mais utilizados é a possibilidade de fornecer continuidade do cuidado e acesso remoto aos dados do Registro Eletrônico em Saúde (RES). Para que isto funcione, é necessário fornecer garantias de que o conteúdo do RES seja compreensível e interpretado de maneira semelhante por qualquer profissional em qualquer situação, i.e. interoperabilidade semântica.

Ao nível de S-RES, isso pode ser alcançado com o uso de vocabulários e terminologias clínicas (4). Alguns exemplos são a Classificação Internacional de Doenças v.10 (CID-10) (5), a Classificação Internacional de Atenção Primária v.2 (CIAP-2) (6) e a SNOMED-CT (7). No cenário brasileiro, CID-10 e CIAP-2 já são utilizadas. A SNOMED-CT está em fase de tradução para incorporação, com a possibilidade de ser utilizada de forma mais abrangente. Porém, este processo pode ser um processo demorado (8).

Como forma de viabilizar a interoperabilidade semântica, uma abordagem bastante utilizada é 0 desenvolvimento e aplicação de mapeamentos entre terminologias e 
vocabulários. Segundo Chan e Zeng (9), o mapeamento consiste em achar termos ou identificadores semanticamente equivalentes (ou aproximados) em terminologias e/ou vocabulários diferentes para descrever um dado conceito. O mapeamento pode ser construído com o auxílio de especialistas ou com auxílio de estratégias computacionais.

A utilização de mapeamentos entre terminologias, por exemplo, em um SRES, pode auxiliar tanto na identificação de termos novos, quanto como meio para proposição de termos de fontes diferentes. Essa tarefa pode auxiliar tanto no enriquecimento semântico do RES, quanto no fornecimento de meios para a análise e interpretação de registros (10). Ainda, pode auxiliar em outra área importante, especificamente a busca e recuperação de conteúdo em RES (11).

Apesar disso, tanto os mapeamentos quanto as terminologias precisam ser mais exploradas no Brasil (12). Isso pode ser percebido e.g. em Segundas-Opiniões Formativas (SOFs). SOFs são perguntasrespostas baseadas em evidências científicas geradas a partir de dúvidas derivadas de teleconsultorias (13).

Assim como no RES, as SOFs devem apresentar termos provenientes de terminologias (como o CIAP-2 e CID-10) para identificar o conteúdo da questão disponibilizada. Dessa forma, a ausência de conceitos provenientes de vocabulários controlados e terminologias limita a compreensão, a interpretação, e a identificação do conteúdo quando há troca de informações entre S-RES diferentes ou sobre o conteúdo de SOFs.

Com o intuito de auxiliar e promover o uso de terminologias e dos respectivos mapeamentos, o objetivo deste trabalho é apresentar uma ferramenta que permite investigar e explorar os mapeamentos existentes entre CID-10, CIAP-2 e SNOMED-CT, aplicando-os na indicação de termos correlacionados para enriquecimento terminológico em SOFs. Para isto, foram utilizadas SegundasOpiniões Formativas (SOF) sobre hipertensão e diabetes. Dessa forma, é possível verificar as complementaridades entre estas terminologias e indicar novos códigos para uma ou mais SOFs.

\section{Métodos}

SOFs foram recuperadas e armazenadas, em um banco de dados, de forma programática a partir do portal "BVS Atenção Primária em Saúde" (13). Neste portal são disponibilizadas SOFs de forma pública e gratuita.

A consulta para recuperação de SOFs foi realizada em agosto de 2019, utilizando os descritores 'diabetes' e 'hipertensão' e abrangendo os períodos de publicação disponíveis, de 2007 até 2019. Foram desconsideradas as SOFs que não apresentavam códigos CID-10 ou CIAP-2.

Cada SOF armazenada no portal possui diversos campos, como: (i) Identificador (id); (ii) pergunta; (iii) 
resposta; (iv) Descritores em Ciências da Saúde (DeCS) (14); (v) Referências; (vi) Categoria profissional; (vii) Núcleo de Telessaúde; (viii) CID-10; e (ix) CIAP-2.

A busca das SOFS e o processo de captura dos dados foram realizadas utilizando Web scraping na página do portal BVS APS. Web scraping é uma técnica utilizada para a extração de dados de interesse em um determinado website. (15).

O Web scraping foi realizado utilizando a linguagem JavaScript e a biblioteca cheerioJs (16). De cada SOF disponível, foram armazenados o seu número de identificação (i.e. id), a pergunta e o seu código CIAP-2 e/ou CID-10. Neste momento, foi decidido não utilizar os DeCS no mapeamento por ele não ser tão direcionado ao contexto clínico, mas a questões de indexação e busca na literatura.

As SOFs recuperadas foram armazenadas utilizando como base 0 modelo não-relacional (NoSQL), gerenciado pelo MongoDB. Este modelo foi escolhido pela simplicidade e flexibilidade possibilitada no armazenamento de dados. Isso facilita a construção de uma base de dados em que os dados não são necessariamente uniformes como a que foi construída (17). O MongoDB armazena os dados no formato de JavaScript Object Notation (JSON) e permite que os documentos possam variar de forma bastante flexível.
A partir desta base de dados de SOFs, foi aplicado primeiramente a tabela de mapeamentos entre CIAP-2 e CID-10, utilizando como base 0 arquivo disponibilizado pela Sociedade Brasileira de Medicina de Família e Comunidade (SBMFC) (6) (versão 16 de agosto de 2016).

Em seguida, os registros armazenados foram analisados quanto aos códigos existentes da CIAP-2 na base de dados de SOF; e, verificada a existência de um mapeamento entre esses códigos e a CID-10. Para cada um dos códigos CIAP2 identificados nas SOFs, foram armazenados um ou mais (1-N) códigos da CID-10.

Isso ocorre pelo fato de existir mais códigos indicados como possíveis mapeamentos para CID-10 a partir da CIAP-2. Por exemplo, o código CIAP-2 K87 'Hipertensão com complicações' apresenta 05 mapeamentos para códigos da CID-10, dentre os quais 111 'Doença Cardíaca Hipertensiva' e 115 'Hipertensão Secundária'.

De forma semelhante, foi feito o mapeamento entre os termos CID-10 obtidos no passo anterior com a SNOMEDCT. As complementaridades entre esses dois foram verificadas pela tabela de mapeamentos já disponível na Unified Medical Language System (UMLS), na sua última versão de 2019AB (18).

O mapeamento entre CIAP-2 e SNOMED-CT, neste trabalho, foi derivado do mapeamento realizado em etapa 
anterior, i.e. CIAP-2 $\leftrightarrow$ CID-10 $\leftrightarrow$ SNOMED CT.

\section{Resultados}

A seguir, descreveremos os resultados derivados do desenvolvimento deste trabalho.

\subsection{Web Scraping}

De forma geral, foi analisado o HTML das páginas, buscando as perguntas e seu id. A partir disto, o link das perguntas foi armazenado e posteriormente salvo em um JSON (JavaScript Object Notation). JSON é um formato leve utilizado para troca de dados simples, constituído basicamente de atributos de nomes e valores (19). O passo-a-passo do Web scraping está descrito na figura 1.

Figura 1- Web Scraping.

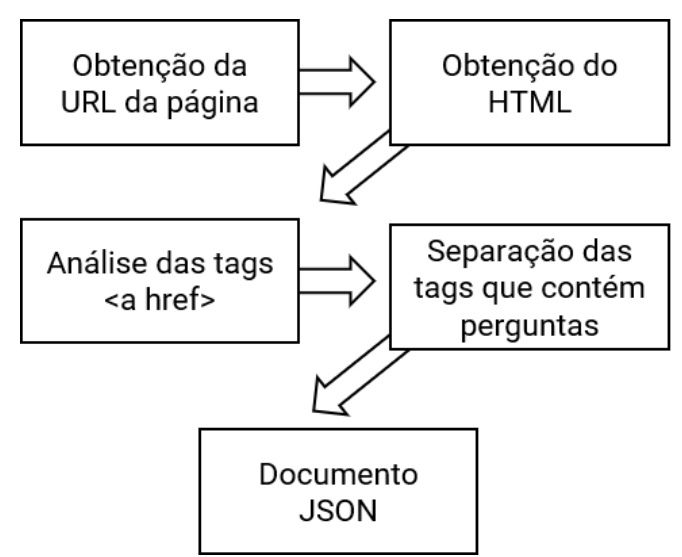

Fonte: Autores.

\subsection{Recuperação e armazenamento de das SOFs}

O passo seguinte compreende a criação de um documento no MongoDB (20) para cada SOF capturada anteriormente. Cada documento possui campos próprios e atributos, conforme descrito na Figura 2.

Figura 2- Documento SOF.

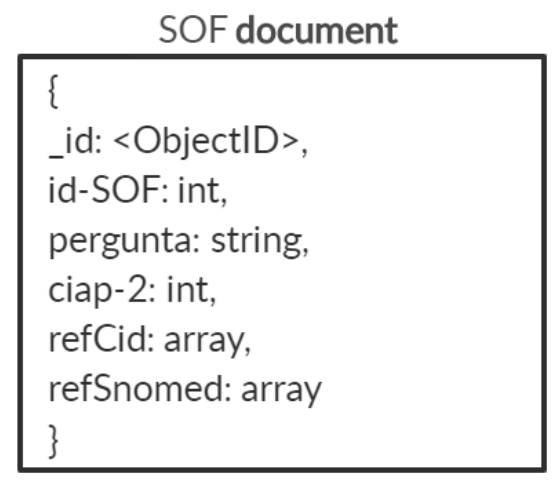

Fonte: Autores

Cada um dos campos pode possuir tamanhos diferentes, visto que, por exemplo, uma determinada pergunta pode estar codificada com apenas um código ou mais de dez códigos CIAP-2. No próprio banco das SOFS são feitas referências aos documentos dos outros bancos, referentes a CID-10 e SNOMED-CT, tornando fácil obter o mapeamento posteriormente.

Da realização da consulta sobre hipertensão e diabetes, foram recuperadas 208 SOFs. Dessas:

- 83 referentes a hipertensão; e

- 125 referentes a diabetes.

Com as SOFs obtidas, foi realizado um processo de filtragem dos dados. Isso foi necessário para identificar as SOFs que apresentavam interseção, em que uma dúvida codificada na área de diabetes pode também ser pertinente para a área da hipertensão, e vice-versa. 
Em seguida, restaram 168 SOFs com identificadores únicos. Deste total, foi retirada uma SOF por ela não possuir código CIAP-2, inviabilizando 0 mapeamento (Figura 3).

Figura 3- Obtenção das SOFS.

\section{SOFS Obtidas do portal}

BVS

168 SOFS com

id's únicos

167 SOFS

Fonte: Autores.

Analisando inicialmente o banco de SOFs foram encontrados ao todo 72 códigos CIAP-2 únicos. Desses, o mais frequente foi 'T89 Diabetes insulinodependente' (Gráfico 1).

Dentre os 72 códigos CIAP-2 únicos, 19 não possuíam um mapeamento para a CID-10, conforme o documento de mapeamentos da SBMFC (6). Isto inclui códigos de procedimentos, em que podemos citar a 'A45 Educação em Saúde', que não está presente em mapeamentos oficiais.

\subsection{Arquitetura da solução para sugestão} de mapeamentos

Além do BD de SOFS, foram construídos mais outros. A saber:

- CID-10;

- Mapeamentos CID-10 e CIAP-2 (6); e
- Mapeamentos CID-10 e SNOMED CT (17).

Utilizando as características do próprio MongoDB, a sugestão de mapeamentos foi construída a partir de referências às SOFs armazenadas. Foi utilizado para isso os campos detalhados anteriormente na Figura 2, i.e.

- refCid, que faz referência ao banco da CID-10 e

- refSNOMED, que faz referência ao banco SNOMED.

O resultado disto é um arquivo JSON contendo todos os mapeamentos recuperados entre os dois bancos, ou podendo também ser detalhada para consultas específicas, e.g o mapeamento para uma pergunta e/ou código específico.

A arquitetura resultante dessa estratégia de mapeamentos pode ser observada na Figura 4.

Figura 4- Arquitetura da solução.

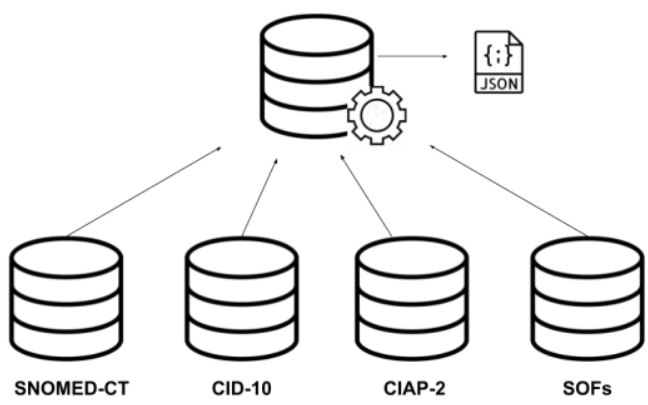

Fonte: Autores. Ícones retirados do Flatlcon.

\subsection{Estudo de caso com mapeamentos}

Um exemplo de indicação de mapeamento proveniente do script pode ser observado na Figura 5. 
Figura 5- Exemplo de mapeamento.

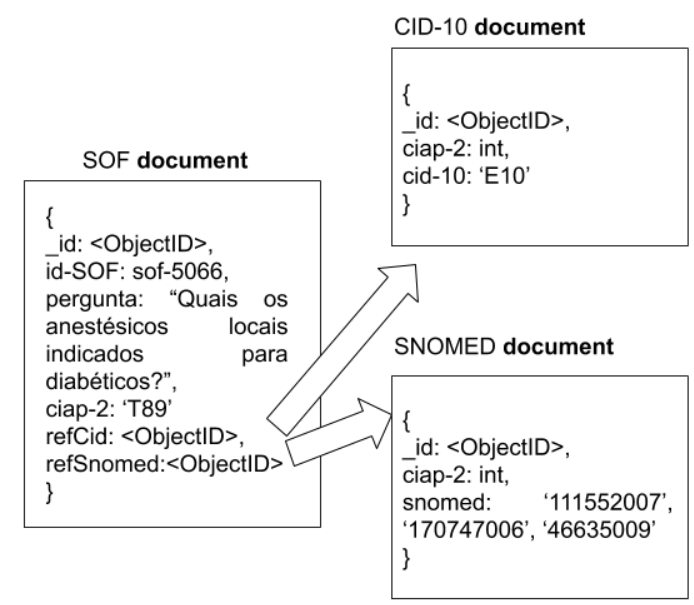

Fonte: Autores.

Esse exemplo contempla para uma pergunta disponibilizada originalmente no portal BVS APS e classificada para diabetes, com um código T89 da CIAP-2.

Especificamente, nos campos refCid e refSnomed estão apontados seus mapeamentos, como, por exemplo, 'T89 Diabetes insulino dependente' da CIAP-2. Este código pode ser mapeado para 'E10 Diabetes mellitus insulino dependente' e 'O24.0 Diabetes mellitus pré-existente, insulino dependente' na CID-10. Já estes da CID-10 podem ser mapeados (de acordo com a UMLS) para diversos outros no SNOMED-CT. Alguns exemplos são ‘111552007 - Diabetes mellitus without complication', '170747006 - Diabetic on insulin', '46635009 - Type 1 diabetes mellitus', entre outros.

\section{Discussão}

Pesquisas sobre 0 desenvolvimento de mapeamentos de terminologias e vocabulários de saúde tornaram-se um tópico bastante importante e pesquisado nas últimas décadas, reforçando a importância de se garantir uma interoperabilidade entre S-RES (21). No Brasil, este campo também tende a crescer tendo em vista as medidas que o governo tem tomado acerca do uso de padrões na saúde, como a Portaria GM/MS no 2.073 de 2011 (22). A literatura mostra também que já estão sendo desenvolvidas metodologias para o mapeamento entre estes padrões da saúde (23), bem como há norma para tal (26).

Neste trabalho, foi mapeado inicialmente apenas dois módulos das terminologias citadas, em áreas referentes a hipertensão e diabetes. Esses módulos consistiram nos subconjuntos por serem áreas importantes para o contexto da atenção primária no Brasil (24). Dessa forma, este módulo poderá ser utilizado quando eventualmente a SNOMED CT for traduzida e incorporada em contexto de produção no Brasil.

Assim, é factível considerar a implementação dessa abordagem, visto que os códigos e termos das SOFs continuarão os mesmos. Nesse caso, da evolução de mapeamentos e inclusão de novos termos, será necessário atualizar (apenas) as tabelas de definição de mapeamentos.

$\mathrm{Na}$ prática, a incorporação do mecanismo de mapeamentos pode ser facilitada pelo desenvolvimento de servidores de terminologias (25). A princípio, a construção do mapeamento 
não considera este fator. Porém, isto poderia ser feito através de uma Interface de Programação de Aplicação (API).

$\mathrm{Na}$ literatura em língua portuguesa, não foram identificadas abordagens que explorem os mapeamentos semelhantes ao que propomos neste trabalho, i.e., utilizando os padrões CIAP-2, CID-10 e SNOMED-CT. Dado que os mapeamentos existentes são vão além da hipertensão e diabetes, outros módulos de conceitos e problemas na saúde podem ser mapeados de forma similar à desenvolvida neste trabalho.

Do ponto de vista da implementação, é importante ressaltar que o próprio MongoDB oferece suporte a consultas em algumas linguagens, como o JavaScript e o Python, bastante utilizadas atualmente. Isso facilita a construção de consultas e a incorporação direta por outros trabalhos.

Apesar dos resultados alcançados, o trabalho apresenta algumas limitações. Uma delas é a avaliação do desempenho da solução desenvolvida num cenário mais amplo, i.e. S-RES ou servidor de terminologia. Isto seria imprescindível para uma possível implementação dos códigos gerados no mapeamento em documentos clínicos.

Outro aspecto é sobre a utilização do DeCS. Se este for utilizado juntamente com técnicas para análise de dados, sugestão de termos ou interpretação automatizada, aliando abordagens de Inteligência Artificial (IA) e Processamento de Linguagem Natural (PLN), pode se transformar em uma abordagem interessante futuramente.

Uma questão que é importante ressaltar está relacionada a qualidade e a possibilidade de erros nos mapeamentos. Não é objetivo deste trabalho analisar a qualidade dos mapeamentos constituídos e disponibilizados na literatura, mesmo considerando a aplicabilidade dos mesmos para finalidades diferentes, e.g. diagnóstico e/ou relato pelo paciente com a CID-10 e a CIAP-2, respectivamente. Nesse sentido, consideramos que a construção e a exploração de terminologias e mapeamentos devem seguir padrões como a ISO/TR 12.300 (26)

Além disso, os conceitos devem ser submetidos a inspeção formal a partir da análise e interpretação semântica de cada conceito ou classe. $O$ fato de utilizar mapeamentos existentes (num contexto prático) poderá levar a necessidade de realizar adequações.

Todavia e em trabalho anterior com ontologias e dados provenientes da literatura biomédica, foi possível identificar que um conceito de uma terminologia, ou classe de uma ontologia, podem denotar interpretações específicas (10). Em outras palavras, um conceito em um registro de banco de dados (assim como o CID-10 ou CIAP-2 em uma SOF) pode fugir a intenção dos editores (da SOF ou do RES) quando não há uma semântica formal expressa na terminologia ou vocabulário 
empregado. A CID-10 e a CIAP-2 são susceptíveis.

Com os mapeamentos em questão, a SNOMED CT é menos suscetível a este problema, dado que ela utiliza a semântica formal da Ontology Web Language (OWL) para descrever como cada conceito deve ser expresso e interpretado (27). Para minimizar este efeito, foram utilizadas referências nacionais e internacionais, como as recomendações CID-10 e CIAP-2 provenientes da SBMFC; e, os mapeamentos da CID-10 e CIAP-2 com a SNOMED CT descritos na UMLS.

\section{Conclusões}

Neste trabalho, foi desenvolvida uma solução para prover termos CID-10, CIAP2 e SNOMED CT a partir da exploração de mapeamentos entre as fontes terminológicas. Os mapeamentos entre CIAP-2, CID-10 e SNOMED CT foram empregados a partir de documentos recuperados de entidades de referência, especificamente a SBMFC e UMLS.

Com o auxílio da solução apresentada, os mapeamentos existentes foram empregados sobre SOFs sobre hipertensão e diabetes codificadas com a CIAP-2. Finalmente, foi possível recuperar códigos provenientes da SNOMED-CT de acordo com os mapeamentos existentes.

Do ponto de vista prático, este tipo de abordagem viabiliza futuras codificações de SOFs. Isso pode derivar a sugestão de códigos CID-10 e SNOMED
CT na medida em que elas são codificadas com seus respectivos códigos CIAP-2.

Ou seja, disponibilizamos uma abordagem simplificada e que pode vir a ser incorporada em S-RES, explorando outros mapeamentos CID-10, CIAP-2 e SNOMED CT descritos na literatura. Isso pode auxiliar a S-RES que ainda não incorporam a SNOMED CT, por exemplo, a iniciar o processo de codificação e indexação para fins de interoperabilidade semântica futura.

Finalmente, trabalhos futuros são necessários para avaliar a expansão para além da hipertensão e da diabetes, expandindo os módulos traduzidos para outras áreas da atenção primária. Isso facilitaria a utilização dos mecanismos de codificação em S-RES que utilizam o CID10, CIAP-2, SNOMED CT ou qualquer variação dessas três fontes.

- Conflito de interesse: Todos os autores declaram não haver conflitos de interesse ou interesse pessoal/comercial.

- Disponibilidade: http://purl.org/sofs 


\section{Referências}

1. International Organization for Standardization - ISO. ISO/TR 20514:2005 Health informatics Electronic health record - Definition, scope and context. 2005.

2. Araujo T; Pires S; Bandiera-Paiva

P. Adoção de padrões para Registro Eletrônico em Saúde no Brasil. Revista Eletrônica de Comunicação, Informação e Inovação em Saúde, [S.I.], v. 8, n. 4, dec. 2014.

3. Cavalini L; Ahiadzro N; Cook T; Os Registros Eletrônicos em Saúde e seus potenciais impactos no campo da saúde pública. Jornal Brasileiro de Telessaúde vol 2 nํ 4. 2013.

4. Watkins T; Lundberg C; Brokel J; Wilson M; Hardiker N; Terminology use in electronic health records: Basic principles. Urologic nursing. 29. 321-6. 2009.

5. Organização Mundial de Saúde. Classificação Internacional de Doenças v10 (CID-10). 2016.

6. Sociedade Brasileira de Medicina de Família e Comunidade. Classificação Internacional de Atenção Primária v2 (CIAP-2). 2010.

7. SNOMED International. SNOMEDCT [acesso em outubro de 2019]. Disponível em: http://www.snomed.org/ 8. Galvão M; Ricarte I. A SNOMED CT e os Sistemas de Informação em Saúde. Infohome [Internet]. Londrina: OFAJ. 2013.

9. Chan L; Zeng M. Ensuring Interoperability among Subject Vocabularies and Knowledge. 68th IFLA Council and General. Conference. August 18-24, 2002.

10. Da Silva F; Jansen L; Freitas F; Schulz S. Ontological interpretation of biomedical database content. Journal of Biomedical Semantics, 8(1), 24. 2017. 11. Natarajan K; Stein D; Jain S; Elhadad N. An analysis of clinical queries in an electronic health record search utility. Int J Med Inform. 79(7):515-522. 2010.

12. Maciel D; Ferreira D; Marin H. Padrões de terminologias nacionais para procedimentos e intervenções na saúde.
Rev. Adm. Saúde - Vol. 18, № 71, abr. jun. 2018. 2018.

13. BVS Atenção Primária em Saúde [Internet]. O que é SOF? [acesso em outubro de 2019]. Disponível em: https://aps.bvs.br/segunda-opiniaoformativa/.

14. BVS DECS Descritores em Ciências da Saúde [Internet]. O que é? [acesso em outubro de 2019]. Disponível em:

http://decs.bvs.br/P/decsweb2019.htm

15. Cording P; Algorithms for Web

Scraping. Technical University of Denmark. 2011.

16. CheerioJs. CheerioJs [acesso em outubro de 2019]. Disponível em: https://github.com/cheeriojs/cheerio 17. Han J; Haihong E; Le G; Du J. Survey on NoSQL database, 2011 6th International Conference on Pervasive Computing and Applications, Port Elizabeth, 2011, pp. 363-366. 2011. 18. U.S National Library of Medicine [Internet]. Unified Medical Language System (UMLS) [acesso em outubro de 2019]. Disponível em:

https://www.nlm.nih.gov/research/umls/ind ex.html

19. JSON. Introdução ao JSON [acesso em março de 2020]. Disponível em: https://www.json.org/json-pt.html 20. MongoDB Inc. MongoDB [acesso em março de 2020]. Disponível:

https://www.mongodb.com/

21. Cardillo E. Mapping between international medical terminologies. Annex 4 to SHN D3.3. 2015.

22. Brasil - Ministério da Saúde. Portaria № 2073, de 31 de agosto de 2011. 2011. 23. Dias T. Método para mapeamento entre terminologias em saúde, visando a interoperabilidade entre sistemas de informação [dissertação]. São Carlos: Universidade de São Paulo, Bioengenharia; 2014.

24. Lobo L, Canuto R, Dias-da-Costa J, Pattussi M. Tendência temporal da prevalência de hipertensão arterial sistêmica no Brasil. Cad. Saúde Pública [Internet]. 2017.

25. Metke-Jimenez A; Steel J; Hansen D; Lawley M. Ontoserver: a syndicated terminology server. Journal of Biomedical 
Semantics volume 9, Article number: 24 .

2018.

26. International Organization for

Standardization - ISO. ISO/TR 12300:2014 Health informatics -

Principles of mapping between

terminological systems. 2014.

27. SNOMED International. SNOMED CT

Release File Specifications [acesso em

março de 2020]. Disponível em:

https://confluence.ihtsdotools.org/display/

DOCRELFMT/SNOMED+CT+Release+Fil

e+Specifications

Rev. Saúde Digital Tec. Educ., Fortaleza, CE, v. 5, n. 1, p.95-107, jan./abr. 2020.

ISSN: 2525-9063 


\section{Anexos}

Gráfico 1: Termos CIAP-2 mais recorrentes.

Termos CIAP-2 mais recorrentes

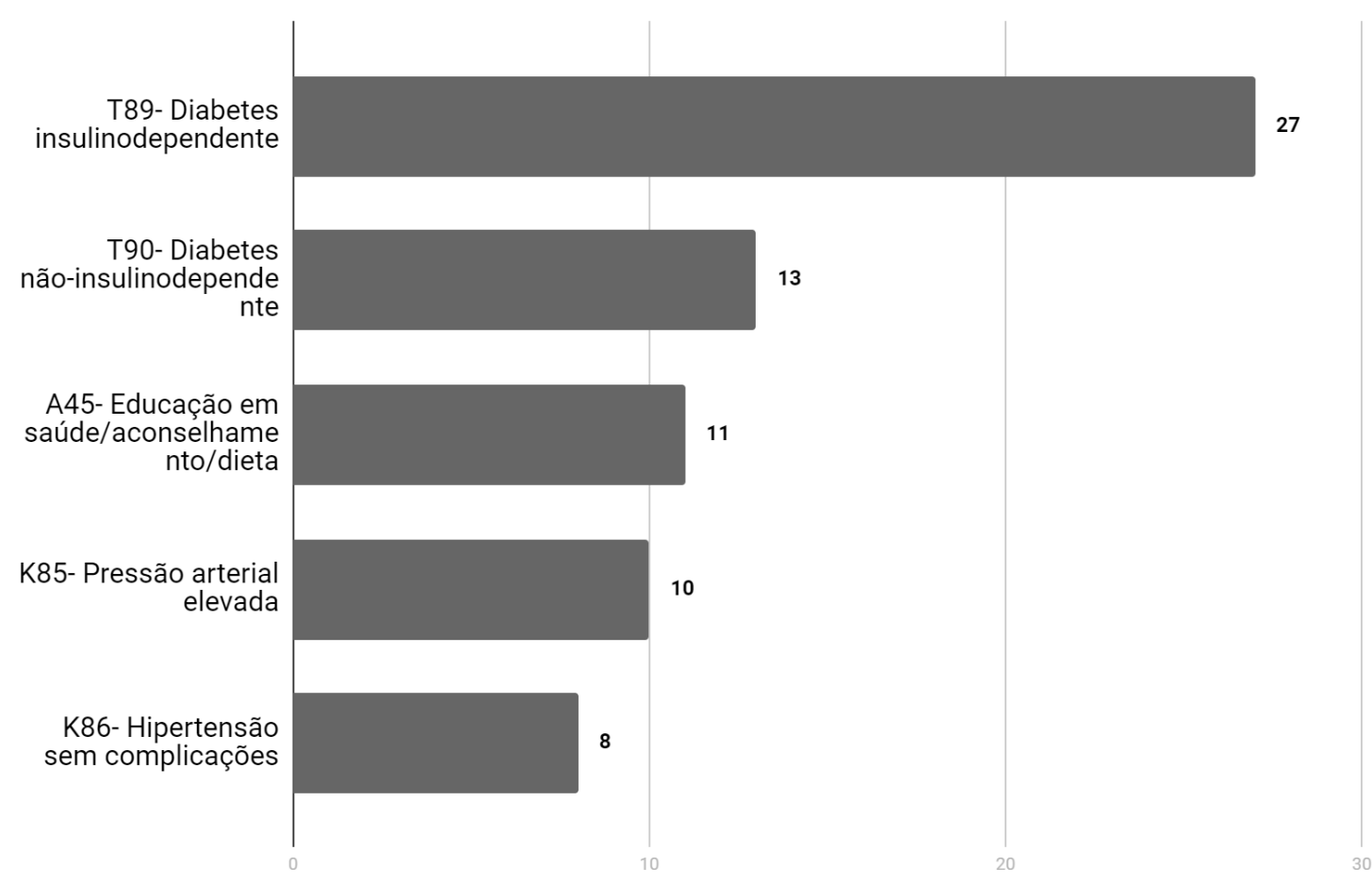

$\mathrm{N}^{\circ}$ de documentos

Fonte: Autores.

Rev. Saúde Digital Tec. Educ., Fortaleza, CE, v. 5, n. 1, p.95-107, jan./abr. 2020.

ISSN: 2525-9063 


\section{Como citar este artigo}

Santos BGT dos; Bettoni GN; Silva FS da. Aplicação de mapeamentos entre termos SNOMED CT, CID-10 e CIAP-2 para enriquecimento terminológico em Segundas-Opiniões Formativas sobre hipertensão e diabetes. Revista de Saúde Digital e Tecnologias Educacionais. [online], volume 5, n. 1. Editor responsável: Luiz Roberto de Oliveira. Fortaleza, mês e ano, p. 95-107. Disponível em: http://periodicos.ufc.br/resdite/index. Acesso em "dia/mês/ano".

Data de recebimento do artigo: 15/10/2019

Data de aprovação do artigo: 25/02/2020

Data de publicação: 17/04/2020 